\title{
Numerical Simulation of the Thin Film Coating Flow in Two-Dimension
}

\author{
Zhiyong Liang, Hui Zhou \\ College of Science, Donghua University, Shanghai, China \\ Email: zhyliang@dhu.edu.cn
}

How to cite this paper: Liang, Z.Y. and Zhou, H. (2017) Numerical Simulation of the Thin Film Coating Flow in Two-Dimension. Open Journal of Fluid Dynamics, 7, 330-339. https://doi.org/10.4236/ojfd.2017.73021

Received: August 4, 2017

Accepted: September 15, 2017

Published: September 18, 2017

Copyright $\odot 2017$ by authors and Scientific Research Publishing Inc. This work is licensed under the Creative Commons Attribution International License (CC BY 4.0).

http://creativecommons.org/licenses/by/4.0/

\begin{abstract}
Thin film coating is a process of making liquid film cover and deposit base body surface by the way of dipping, spraying, sliding or spin coating, which is a kind of modern surface engineering. It plays an important role in the actual processing, such as improving the surface properties, fine processing, and new surface properties. Analysis of the influence of substrating morphology and fluid flow properties itself on coating fluid motion has an important significance to optimize the thin film coating and improve the quality of the final film. The influence from uneven substrate surface's geometry configuration on internal motion of the flow field in slip-coating is analyzed by using the FLUENT software as a calculation platform. A two-dimension model of slip coating under isosceles triangle and isosceles trapezoid substrate was established, and thin film coating fluid motions under different configuration parameters were simulated. It is pointed out that the key factor determining the turbulence generation and evolution is the parameter of substrating surface nature. The effects of the change of Reynolds number on turbulent appearance and action area are studied. The velocity contours of fluid field on different substrate surfaces are shown, and the impact of substrate geometry on the backwater region is analyzed.
\end{abstract}

\section{Keywords}

Numerical Simulation, Thin Film, Slip-Coating, Surface Engineering, Fluid Mechanics

\section{Introduction}

Thin film coating is a kind of modern surface engineering, which generally refers to the process of covering substrate surface with a layer of liquid film. At present, coating technology including electroplating, painting, thermal spraying and va- 
por deposition, compared with the heat treatment, bead welding and other surface engineering, has several advantages such as less constraint conditions, large space of selection technology and material type and so on, and is used in practical engineering more and more widely. It can play out in three ways such as fine processing, optimization of surface properties, and making new surface properties [1]. Specifically, thin film coating can be used to improve the coated substrate surface rust-proof property, anti-corrosion property, abrasions resistance, heat resistance and other characteristics, and has been applied in many fields. In the machinery industry, bridge engineering and electric power industry, thin film coating technique is used to improve micro damage phenomenon of closely matched components [2]; In the field of Aeronautics, film coating technology is often used in aviation engine parts to improve engine efficiency to prolong its service life [3]; In the field of ship protection, application of coating technology can greatly improve the imputrescibility and rust preventing characteristics of shipboard equipment [4].

With the development of material science, liquid thin film begins to take a more and more important role, and consequential quality requirements of the coated thin film are more and more high. In modern industrial applications, thin film coating process needs to meet some special requirements such as: the shape of thin film flow surface is complicated, the fluid coating needs to be carried out in a great disturbance; problem of the actual flow of the film is nonlinear; rheological properties of thin film flow cannot be changed arbitrarily; it should meet the demand of high speed coating industrial production. According to the form of thin film defect, it could be divided into two types, discontinuity and continuity [5].

In the actual production, due to factors of mechanical equipment, operation, production process and fluid properties, coating film maybe have some defects which cannot be completely eliminated such as folds, ripple and bubble [6] [7] [8]. Coating defects will certainly affect the coating quality, thereby affecting the properties of the thin film. As the requirements of coating quality and aesthetic appearance of products continuously increase, how to control or reduce the defects of film coating appearance has become an urgent problem to solve, and this needs to do some numerical simulation in the coating fluid mechanics research. In this paper, research purposes are to analyze the influence of substrating morphology and fluid flow properties itself on coating fluid motion to optimize the thin film coating and improve the quality of the final film.

\section{Coating Fluid Simulation Theory}

In fluid dynamics foreign scholars have carried out much research work on the film flow. However, in this field, most previous studies about the flow of the film focused on the flat surface problem. In recent years, research of the liquid film flow characteristics on surface of basal with specific geometry morphology begins. The reason for this study is the surface of the thin film coating substrate is never perfectly flat but quite complex in the actual production. 


\subsection{Couette Flow Model}

The film flow on smooth surface can be approximated as a shear flow between two infinite flat plates with rigid surface and specific geometry morphology, which is called the Couette flow. This is a basic research model of the thin film coating.

Two-dimension Couette model is shown in Figure 1. The lower flat plate is fixed, the upper mobile flat plate moves at a constant velocity $v$ along the $x$ direction. The velocity distribution between the two flat plates is as follows:

$$
u=\frac{1}{2 \mu} \frac{\mathrm{d} p}{\mathrm{~d} x} y^{2}+c_{1} y+c_{2}
$$

The boundary conditions are as follows:

$$
\left\{\begin{array}{l}
y=0, u=0 \\
y=h, u=v
\end{array}\right.
$$

where $u$ is the distribution velocity, $\mu$ is fluid viscosity, $p$ is the pressure, $h$ is the distance between the parallel plates, $x, y$ represents the spatial coordinates respectively, and $v$ is a constant velocity along the $x$ direction.

The velocity distribution can be generated by substituting Formula (1-2) into Formula (1-1) as follows:

$$
u=\frac{v}{h} y+\frac{1}{2 \mu} \frac{\mathrm{d} p}{\mathrm{~d} x}\left(y^{2}-b y\right)
$$

The velocity distribution of dimensionless form is expressed as

$$
\frac{u}{v}=\frac{y}{h}+D\left(1-\frac{y}{h}\right) \frac{y}{h}
$$

In the above formula, $D$ is the dimensionless pressure gradient

$$
D=-\frac{b^{2}}{2 \mu \nu} \frac{\mathrm{d} p}{\mathrm{~d} x}
$$

\subsection{Calculation Model and the Control Equations}

Thin film coating fluid mechanics problems studied in this paper are based on the improvement of the Couette flow model, in which the bottom surface is undulate substrate. The change of the internal structure of the flow field caused by the uneven geometry is studied. Physical models of the shear drag flow by the

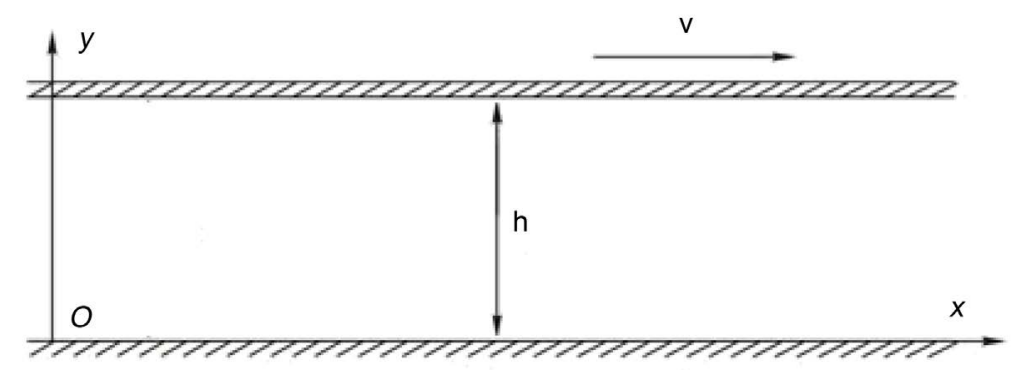

Figure 1. Couette flow diagram. 
approximate Couette model are shown in Figure 2(a) and Figure 2(c), and the calculation models see Figure 2(b) and Figure 2(d). Among them, the top of model is a flat plate which can do translation, and the backplane silhouette is a cyclical fluctuations curve with isosceles triangle or isosceles trapezoid groove. As with the Couette model, the lower base plate is fixed, and the upper flat plate can move at a constant velocity $v$ along the $x$ direction.

Under the hypothesis that the roof and floor are infinite and the base plate geometry is periodic, one circle can be selected for calculation, as shown in Figure 2. Thin film coating fluid in general can be regarded as the Newton fluid, to satisfy the control equation

$$
\begin{gathered}
\frac{\partial \boldsymbol{u}}{\partial t}+\boldsymbol{u} \cdot \nabla \boldsymbol{u}=\boldsymbol{f}-\frac{1}{\rho} \nabla p+\frac{1}{\rho} \nabla(\lambda \nabla \cdot \boldsymbol{u})+\frac{1}{\rho} \nabla \cdot(2 \mu S) \\
\frac{\partial \rho}{\partial t}+\nabla \cdot(\rho \boldsymbol{u})=0 \\
\frac{\partial(\rho T)}{\partial t}+\nabla \cdot(\rho \mathbf{u} T)=\nabla \cdot\left(\frac{k}{c_{p}} \operatorname{grad} T\right)+S_{T}
\end{gathered}
$$

In the formula, $\boldsymbol{u}$ is the velocity vector, $\rho$ is the density, $t$ is the time, $T$ is the temperature, $k$ is heat conduction coefficient, $c_{p}$ is specific heat capacity, and $S_{T}$ is viscous dissipation.

In Figure 2: (a) the physical model with isosceles triangle; (b) the computational model with isosceles triangle; (c) the physical model with isosceles trapezoid groove; (d) the computational model with isosceles trapezoid groove. Usually, Formula (1-5) is called the momentum conservation equation, Formula (1-6) is called the mass conservation equation, and Formula (1-7) is called the

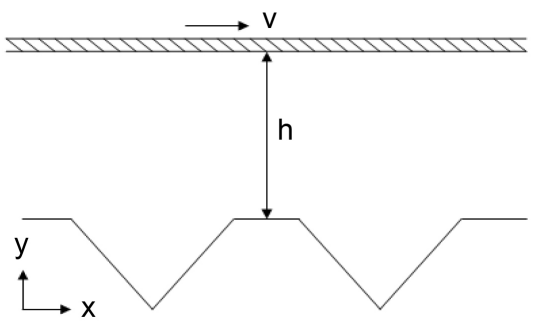

(a)

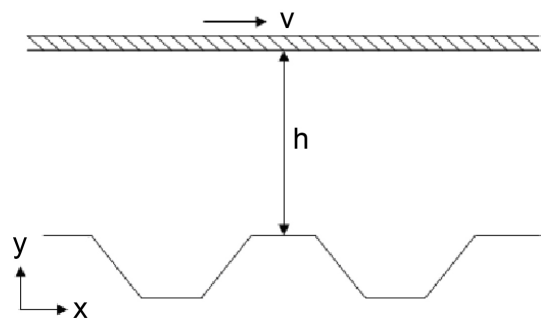

(c)

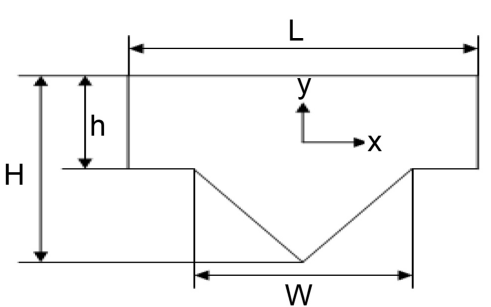

(b)

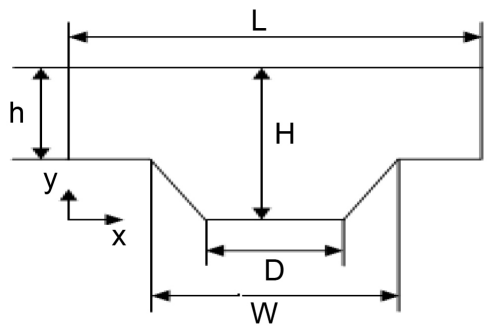

(d)

Figure 2. Physical model and computational model of the backplane silhouette is a cyclical fluctuations curve. 
energy conservation equation. Those formulas constitute equations of Newton fluid motion and are also known as the Navier-Stokes equations. Reference to Couette flow model, on the roof to be applied moving wall boundary condition, the floor uses the solid wall boundary condition.

\section{The Result of Calculation and Simulation}

\subsection{Influence of Roughness of Triangular Base Plate on the Flow Field}

From the calculation results, compared with Couette flow on the flat base plate, with a fixed plate distance $H=1.6$ and constant initial Reynolds number $\operatorname{Re}=10$, substrate irregularity degree is a key factor affecting the formation of eddy current. Seen from Figure 3, with the increase of basal plate roughness, flow separation

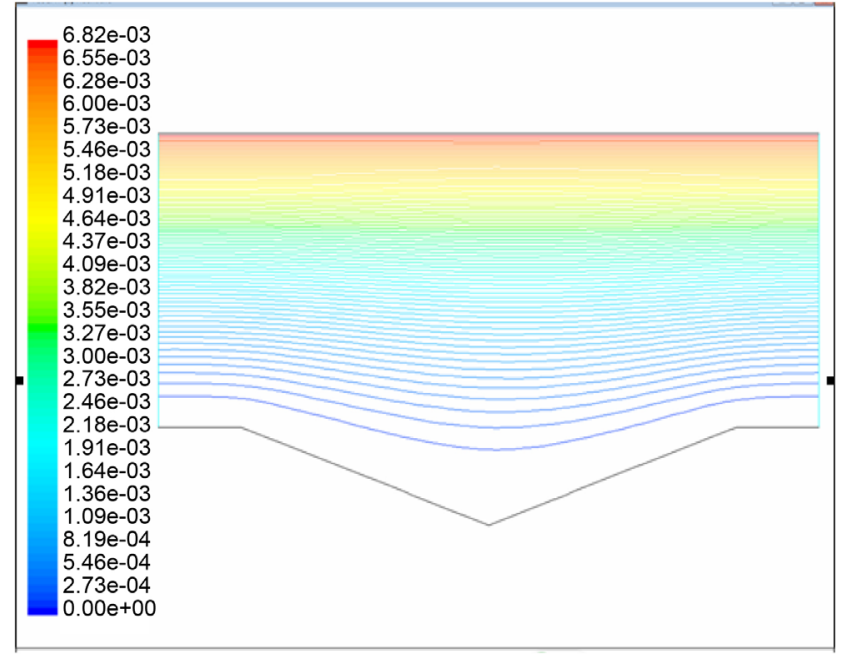

(a)

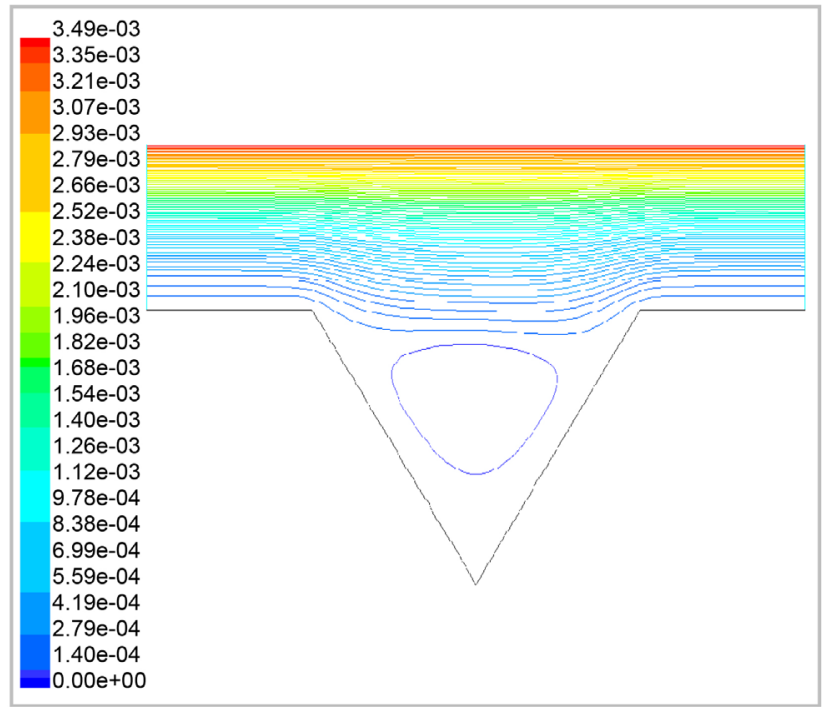

(c)

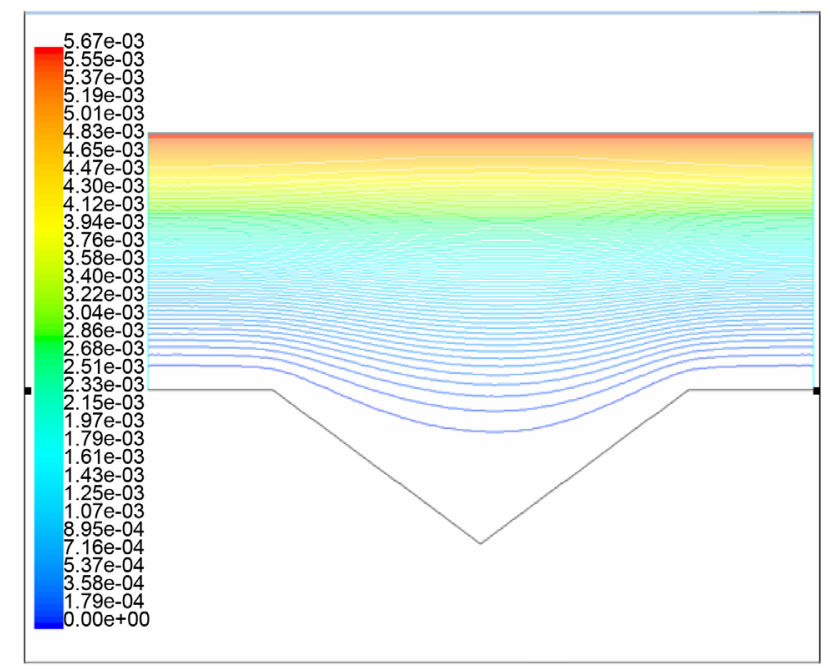

(b)

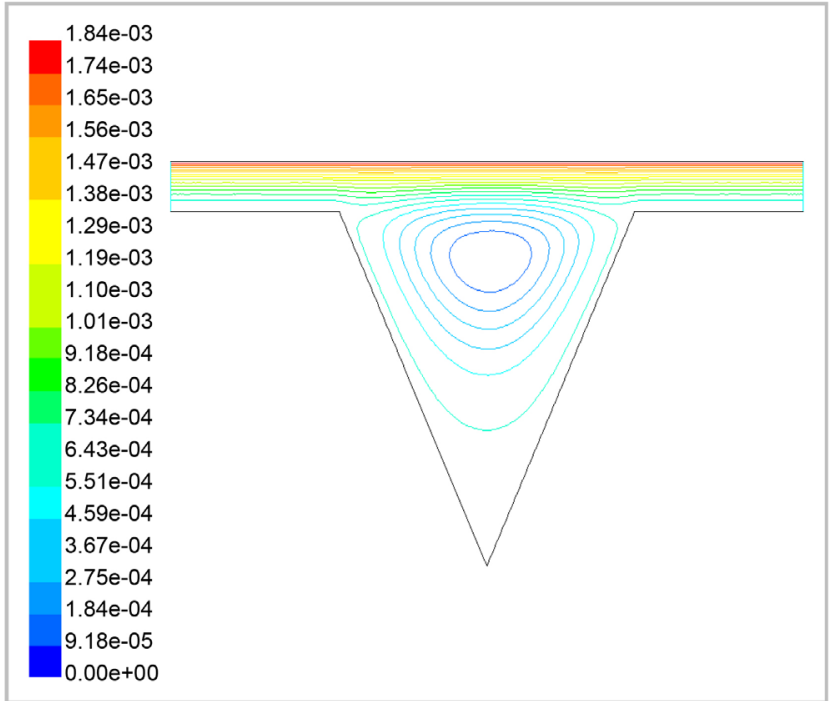

(d)

Figure 3. Stream lines distribution with different roughness $r$ at identical plate distance $H=1.6$ and initial Reynolds number $R e=$ 10 on triangular basal plate. (a) $r=1 / 6$; (b) $r=0.3$; (c) $r=0.625$; (d) $r=1$. 
phenomenon of thin film coating fluid occurs, accompanied by the vortices. Increasing unevenness $r$ will make the eddy current phenomenon more and more obvious, and effect the location of vortex generation. Here defined unflatness of the basal plate with triangular groove is $r=(H-h) / W$.

\subsection{Influence of Roughness of Trapezoid Substrate on the Flow Field}

Thin-film coating flow on trapezoidal base board and triangular basal floor are analogous. Similar with the approximate Couette flow on triangular basal plate, as seen from Figure 4, with the increase of basal plate roughness $r$, flow separation phenomenon of thin film coating fluid field occurs, accompanied with gradual appearing of eddy current. After eddy arising, the increasing of roughness will make the vortex phenomenon more and more obvious. In other words,

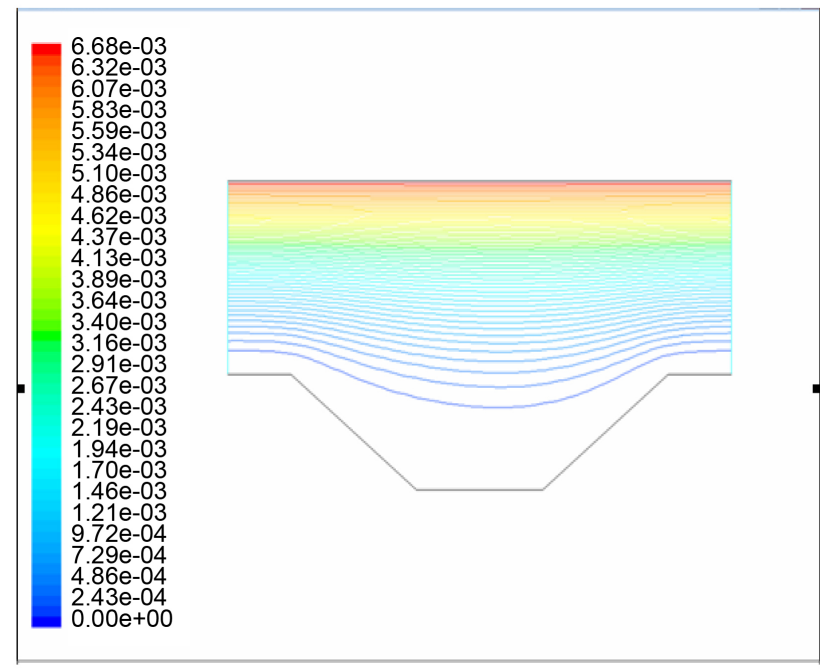

(a)

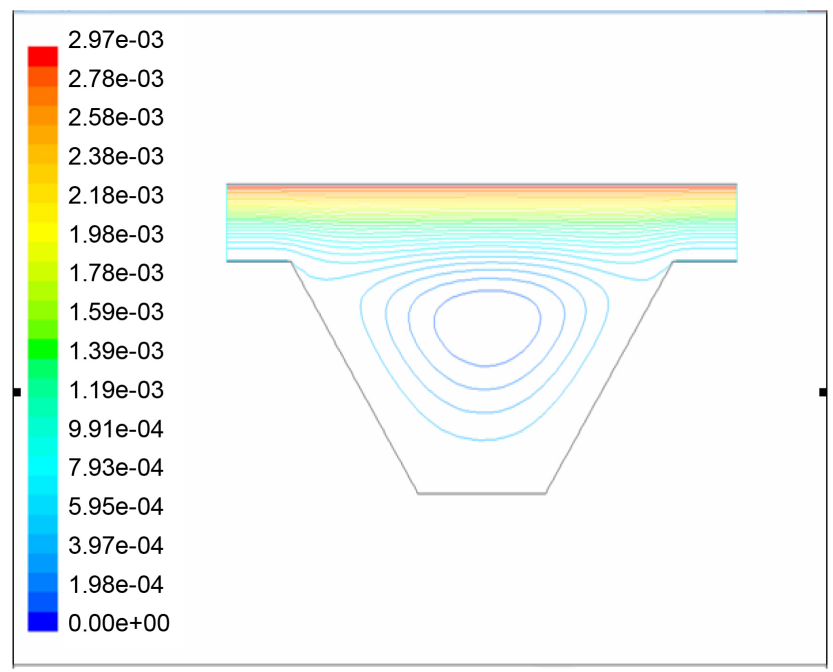

(c)

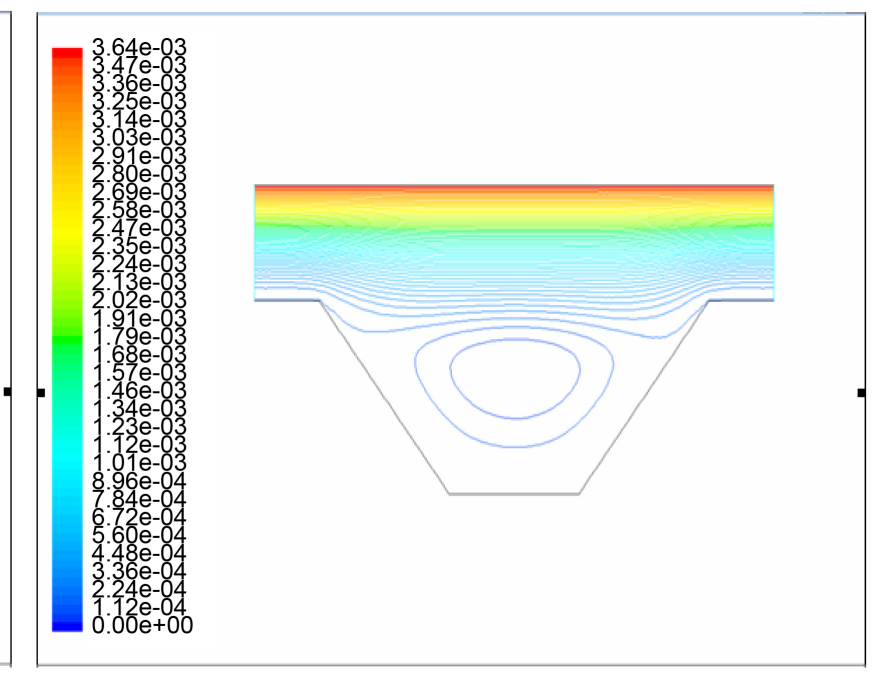

(b)

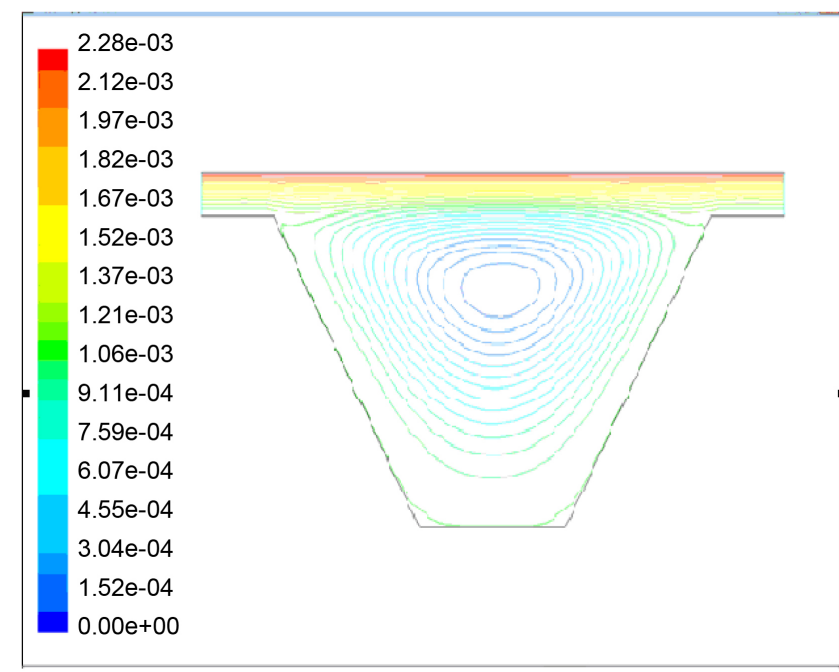

(d)

Figure 4. Stream lines distribution with different roughness $r$ at identical plate distance $H=1.6$ and initial Reynolds number $R e=10$ on trapezoid substrate. (a) $r=3 / 8$; (b) $r=5 / 8$; (c) $r=6 / 8$; (d) $r=7 / 8$. 
increasing the roughness of $\mathrm{R}$ will expand the scope of the vortex. Here the definition of basal plate roughness with isosceles trapezoid is $r=(H-h) /(W-D)$.

\subsection{Analysis of the Influence of Reynolds Number on the Flow Field of the Thin Film Coating}

In theory, film coating fluid should maintain in the laminar or nearly laminar flow state, which means that the flow must be carried out as far as possible at a low Reynolds number range [9]. Low Reynolds number means that the viscous force dominates flow process, in other words, that the effect of inertia force can be ignored to some extent [10]. As can be seen from the Figure 4 and Figure 5 , when the roughness is small such as $r<0.3$, no eddy current arises in low Reynolds number range. State of laminar flow is relatively simple; it's redundant to give details. Here is the only research to produce eddy current flow on the uneven base plate, and this means roughness of the basal plate chosen to discuss is greater than 0.3 . Triangle plate and trapezoidal plate flow are similar, so just one

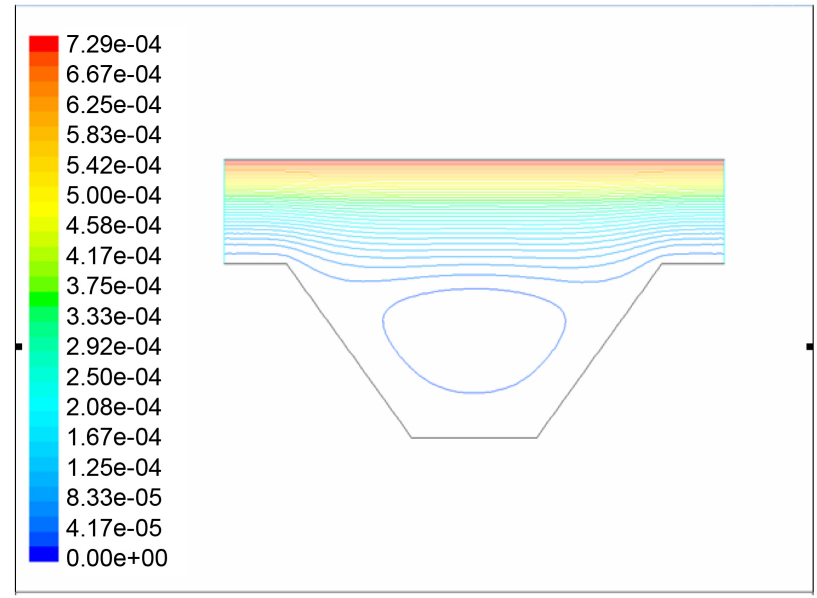

(a)

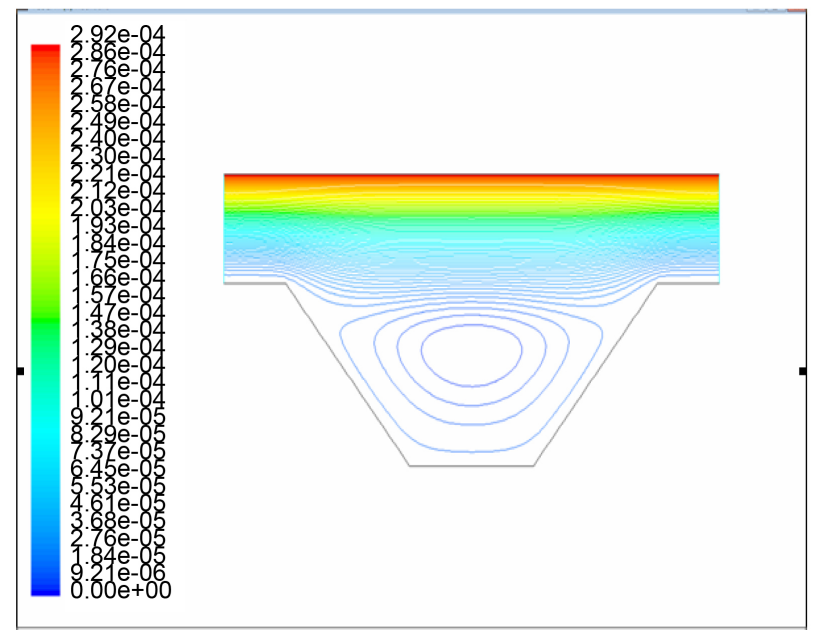

(c)

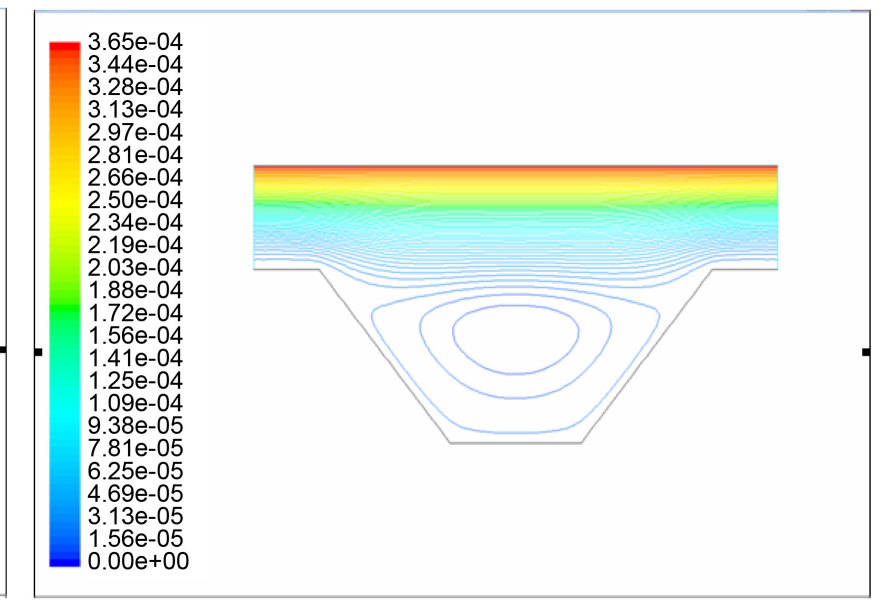

(b)

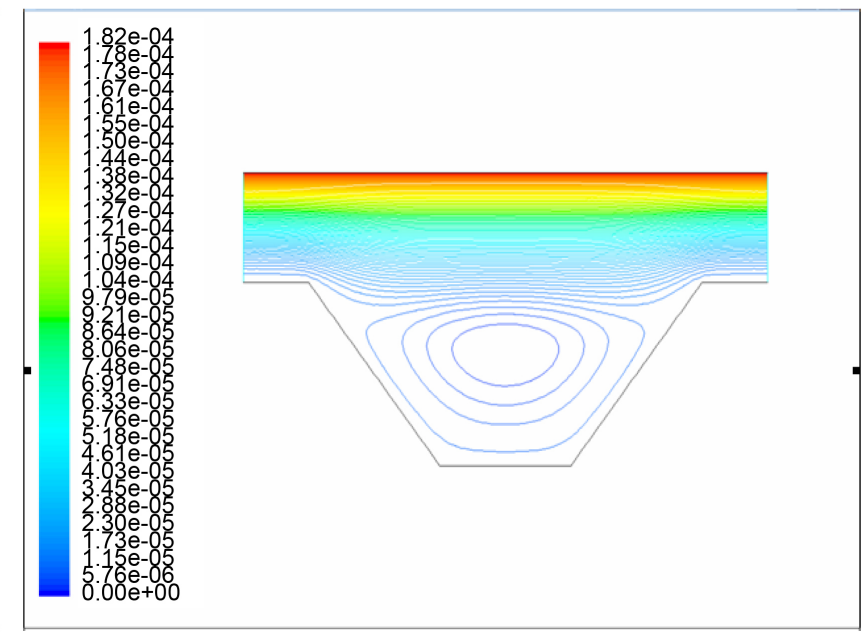

(d)

Figure 5. Stream lines distribution with different Reynolds numbers $\operatorname{Re}$ at unflatness $r=0.6$. (a) $R e=5$; (b) $R e=30$; (c) $R e=60$; (d) $R e=90$. 
of them is chosen to study.

As can be seen from Figure 5, at a low Reynolds range of 1 - 100, when Reynolds number is small, near the basal plate position the volute is small, and flow state is relatively stable. With the increase of Reynolds number, the eddy is more and more obvious to see. But when the Reynolds number increases to a certain extent, volute changes will not be obvious. In other words, compared with the unevenness, the Reynolds number for the eddy current generation and development is not the dominant factor.

\subsection{Analysis of Thin Film Coating Fluid Velocity}

Velocity contours of film coating fluid on the triangle basal plate and trapezoid substrate at the same low Reynolds number are respectively shown as Figure 6 and Figure 7. Here selected to study is the fluid field on basal plate with high roughness that is greater than 0.5 , since there is no need for the research on substrate with a low unevenness (such as $r<0.3$ ) by the reason that the velocity contours of fluid field on substrate at low trapezoid substrate are similar because its state is laminar at low Reynolds number range, while the flow state on the basal plate with larger roughness is turbulent accompanied by segregation phenomenon appearing. As can be seen from the diagram, the fluid velocity close to the bottom is zero, and the overall velocity distribution is symmetrical (in the range of low Reynolds). Whether triangular or trapezoidal substrate, there is an oval center dead zones (backwater region), where the fluid is in static state, and velocity of the surrounding fluid is not equal to zero. Moreover, due to the influence of unevenness, dead zone center arises in a slightly different position, along with the increase of roughness, location has been moved up slightly. There is no center backwater region in the flow field of laminar state, so the center dead zones can be taken as a reference to judge whether the vortex exists or not.

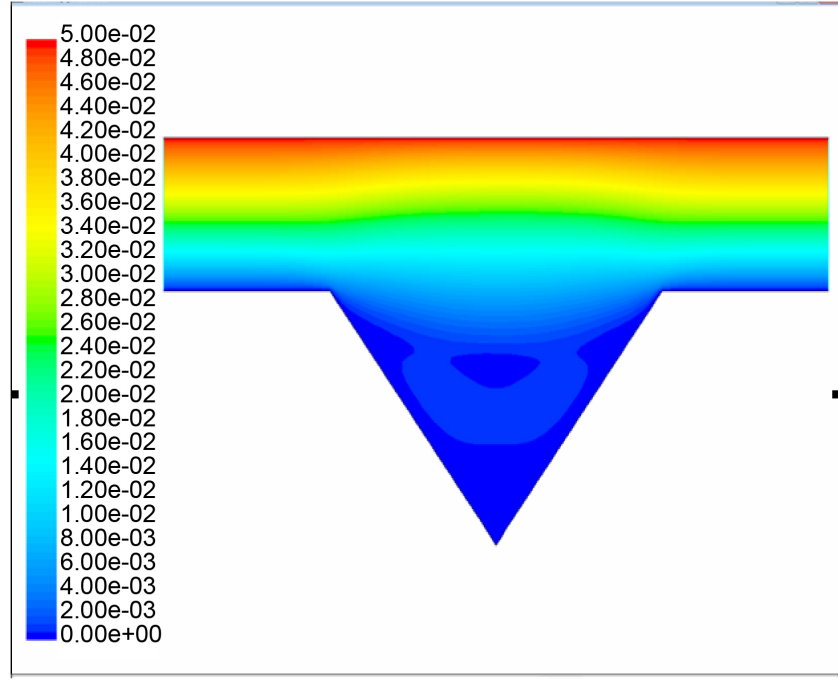

(a)

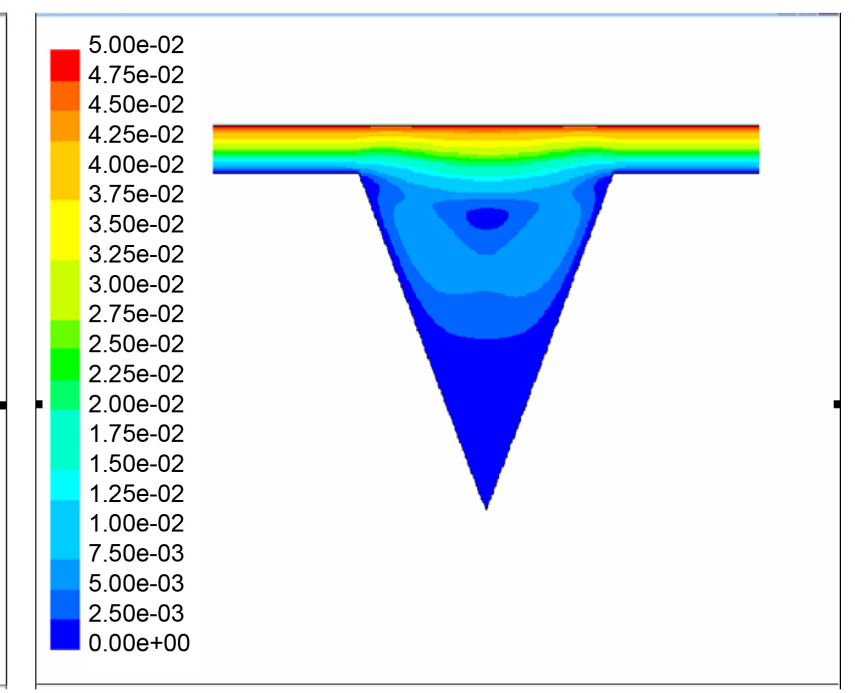

(b)

Figure 6. Contours of the velocity on triangle base plate. (a) $r=5 / 8$; (b) $r=1.0$. 


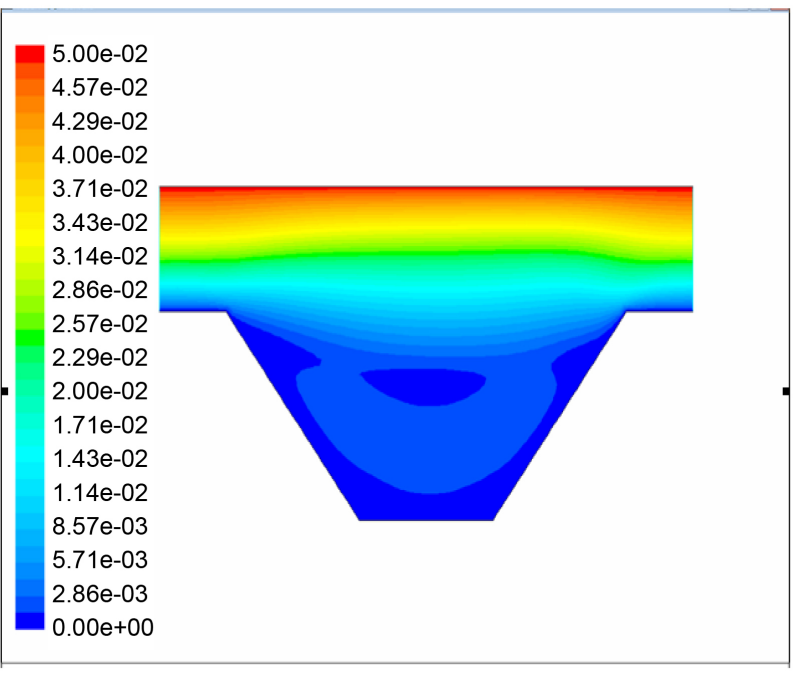

(a) 5.00e-02

$4.57 \mathrm{e}-02$

$4.29 \mathrm{e}-02$

$4.00 \mathrm{e}-02$

$3.71 \mathrm{e}-02$

$3.43 e-02$

$3.14 \mathrm{e}-02$

$2.86 \mathrm{e}-02$

2.57e-02

$2.29 \mathrm{e}-02$

2.00e-02

$1.71 \mathrm{e}-02$

$1.43 \mathrm{e}-02$

$1.14 \mathrm{e}-02$

$8.57 \mathrm{e}-03$

$5.71 \mathrm{e}-03$

$2.86 \mathrm{e}-03$

$0.00 \mathrm{e}+00$

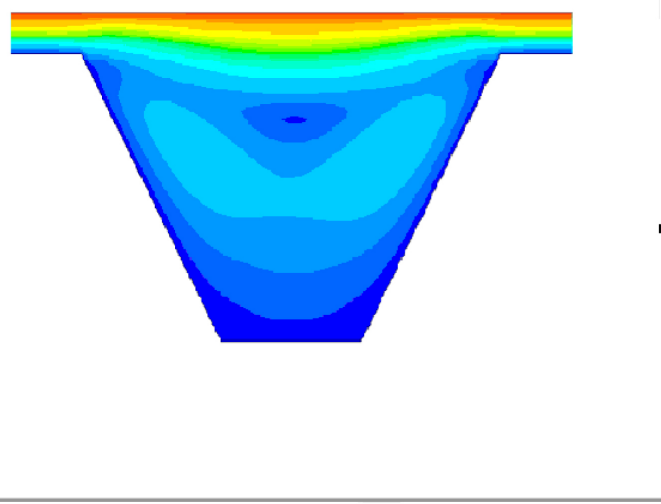

(b)

Figure 7. Contours of the velocity on trapezoid base plate. (a) $r=5 / 8$; (b) $r=7 / 8$.

\section{Conclusion}

We proposed a two-dimension model of slip coating under isosceles triangle and isosceles trapezoid substrate, and thin film coating fluid motions under different configuration parameters were simulated. It is pointed out that the key factor determining the turbulence generation and evolution is the parameter of substratum surface nature, with the increase of basal plate roughness, flow separation phenomenon of thin film coating fluid occurs, accompanied by the vortices. Increasing unevenness $r$ will make the eddy current phenomenon more and more obvious, and effect the location of vortex generation. The effects of the change of Reynolds number on turbulent appearance and action area are studied, compared with the unevenness, the Reynolds number for the eddy current generation and development is not the dominant factor. The velocity contours of fluid field on different substrate surfaces are shown, and the impact of substrate geometry on the backwater region is analyzed. The center dead zones exists can be taken as a reference to judge whether the vortex exists.

\section{Acknowledgements}

This work was partly supported by the Chang Jiang Youth Scholars Program of China and grants (51373033 and 11172064) from the National Natural Science Foundation of China to Prof. Xiaohong Qin. As well as "The Fundamental Research Funds for the Central Universities" and "DHU Distinguished Young Professor Program" to her. It also has the support of the Key grant Project of Chinese Ministry of Education (No 113027A). This work has also been supported by "Sailing Project" from Science and Technology Commission of Shanghai Municipality (14YF1405100) to Dr. Hongnan Zhang.

\section{References}

[1] Jiang, T.-Q. (2004) Chemical Rheology. East China University of Science and 
Technology Press, Shanghai, 223-465.

[2] Wang, L., Mei, X.-Z. and Ma, J.H. (2006) Application of Thermal Spray Technology on Resistance to Fretting. Nonferrous Metals, 93-95.

[3] Fu, J.-B. and Zhou, S.-K. (2006) Application of the Thermal Spraying Technology in Aero-Engine Part and Its Service. Failure Analysis and Prevention, 1, 61-64.

[4] Li, X.-H., AI, Y.-H. and He, X.-J. (2006) Application of Thermal Spray on Ship Self-Defence Field. Mine Warfare \& Ship Self-Defence, 14, 38-41.

[5] Edgar, B.G. and Edward, D.C. (1995) Coating and Drying Defects Troubleshooting Operating Problems. A Wiley-Interscience Publication, New York, 4-10, 75-86.

[6] Liu, Z.-M., Jin, Y.-M. and Liu, H.-M. (2008) Progress on Formation and Prevention of Defects in Thin Film Coating. Journal of Safety and Environment, 8, 135-139.

[7] Taylor, D.J. and Bimie, D.P. (2002) A Case Study in Striation Prevention by Targeted Formulation Adjustment: Aluminum Titanate Sol-Gel Coatings. Chemistry of Materials, 14, 1488-1492. https://doi.org/10.1021/cm010192c

[8] Steven, J.W. and Chen, K.-P. (1999) Large Growth Rate Instabilities in Three-Layer Flow down an Incline in the Limit of Zero Reynolds Number. Physics of Fluids, 11, 3270-3282. https://doi.org/10.1063/1.870187

[9] Scholle, M., Wierschem, A. and Aksel, N. (2004) Creeping Films with Vortices over Strongly Undulated Bottoms. Acta Mechanica, 168, 167-193. https://doi.org/10.1007/s00707-004-0083-4

[10] Wierschem, A. and Aksel, N. (2004) Creeping Films with Vortices over Strongly Undulated Substrates. Physics of Fluids, 12, 58-73.

\section{Scientific Research Publishing \\ Submit or recommend next manuscript to SCIRP and we will provide best service for you:}

Accepting pre-submission inquiries through Email, Facebook, LinkedIn, Twitter, etc. A wide selection of journals (inclusive of 9 subjects, more than 200 journals)

Providing 24-hour high-quality service

User-friendly online submission system

Fair and swift peer-review system

Efficient typesetting and proofreading procedure

Display of the result of downloads and visits, as well as the number of cited articles

Maximum dissemination of your research work

Submit your manuscript at: http://papersubmission.scirp.org/

Or contact ojfd@scirp.org 